\title{
Internal Fixation of Unstable Pelvic Ring Injuries via the Modified Stoppa Approach
}

\author{
TK Ong, MBBS, EH Khoo, MS Orth, Z Osman, MS Orth \\ Department of Orthopaedics and Traumalogy, Hospital Pulau Pinang, Pulau Pinang, Malaysia
}

\begin{abstract}
Background: The modified Stoppa approach was first described in 1993 by Hirvensalo as an alternative to the ilioinguinal approach for treatment of pelvic ring fracture. Methods: This is a retrospective study of 5 patients with unstable pelvic fracture treated with internal fixation using the modified Stoppa approach. Residual displacement of the pelvic ring was measured and graded using the methods described by Matta and Lindahl. Functional outcomes were assessed using the modified Merle D' Aubigne and Postel Hip scoring system. Results: Of all patients with Type C pelvic injury, three had concomitant acetabular fracture. The mean Injury Severity Score (ISS) score was 39. Anatomic reduction of the pelvic ring was achieved in 3 patients and good reduction in 2 patients. Three patients had good functional outcomes. One patient had superficial wound infection. Three patients had erectile dysfunction. Conclusion: The modified Stoppa approach is appropriate for internal fixation of unstable pelvic ring injury.
\end{abstract}

\section{Key Words:}

Unstable Pelvic Fracture, Internal Fixation, Stoppa Approach, Functional Outcomes

\section{INTRODUCTION}

Unstable pelvic ring injury is characterized by a disruption of the posterior sacroiliac arch as well as a rupture of the pelvic floor $^{1}$. It results from high energy trauma, such as motor vehicle accident and fall from a height, and is usually associated with other injuries.

Various surgical approaches have been described previously for internal fixation of the pelvic ring. An ilioinguinal approach, described by Letournel, is widely used as the exposure of choice for restoration of the displaced superior rami fractures as well as fixation of the anterior column and wall fracture of the acetabulum ${ }^{2}$. Often, a second skin incision or an extensile approach is required for fixation of unstable fracture. Possible injuries to the inguinal neurovascular bundle remain a major concern and lateral femoral cutaneous nerve palsies occur frequently.
Hirvensalo and Cole described the modified Stoppa approach as an alternative; the original Stoppa procedure was used to repair difficult abdominal hernias ${ }^{3}$. Using this extra-peritoneal approach through the rectus abdominus muscle, a wide exposure of pelvic ring from the pubic body to the anterior sacroiliac joint can be visualised. Based on the current concept of minimal invasive surgery that emphasizes less tissue dissection, the modified Stoppa approach has gained its popularity. However, it is not widely performed locally. We evaluated 5 patients with unstable pelvic ring fracture treated surgically through the modified Stoppa approach.

\section{MATERIALS AND METHODS}

Five patients who presented with unstable fracture of the pelvic ring between September 2009 and May 2010 were included in this retrospective study.

\section{Operative technique}

All patients were positioned supine on a radiolucent table to achieve unobstructed fluoroscopic views of the anteroposterior pelvic inlet \& outlet. The injured lower limb was draped freely with both hips and knees slightly flexed to relax the iliopsoas muscles. A Foley catheter was inserted to protect the bladder. Prophylactic antibiotic (cefuroxime) and low-molecular weight heparin were administered in all cases. With the surgeon standing on the uninjured side, a Pfannenstiel's skin incision was made about 1 to 2 fingerbreadths superior to the pubic symphysis. The subcutaneous fatty layer was elevated away from the underlying rectus fascia. The rectus abdominus muscle was separated along the linea alba.

Subsequently, the transversalis fascia was opened via blunt dissection to gain access to the pre-peritoneal space. Care was taken to protect the bladder and spermatic cord. The rectus abdominus muscle was partially released near its insertion on the anterior aspect of the pubic body. The anterior abdominal wall was reflected away from the peritoneal sac by inserting a Hoffmann retractor over the superior pubic ramus. A Deaver retractor was used to protect the external iliac vessels. The corona mortis, which is the 


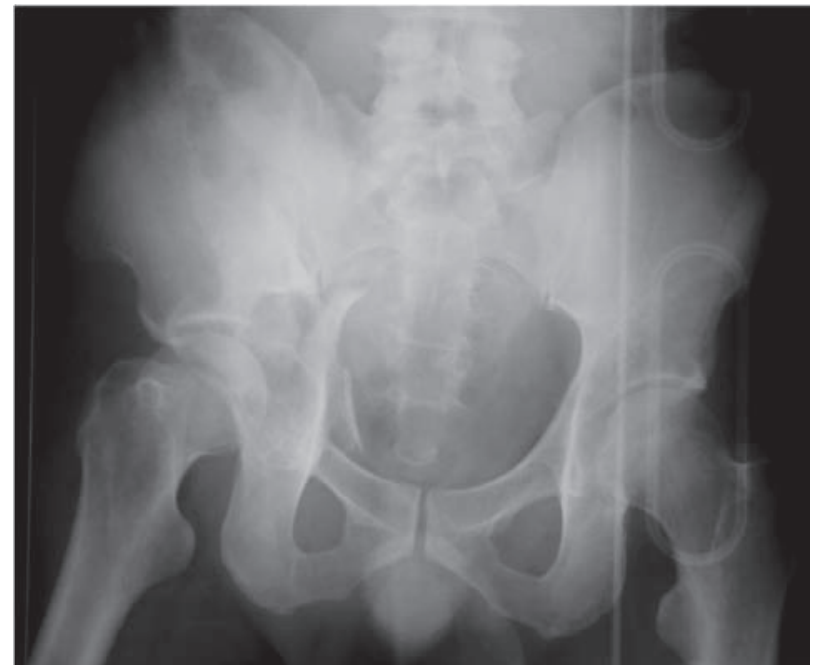

Fig. 1: Preoperative radiograph of the pelvis.

connecting vessel between the obturator and femoral artery, was identified and ligated. Then, the pelvic ring was exposed subperiosteally from the pubic symphysis to the anterior aspect of the sacroiliac joint. A light source from a surgical headlamp or an endoscope was used to illuminate the deeper structures.

Depending on the fracture pattern, further dissection to the quadrilateral surface was performed. The obturator neurovascular bundle was identified on the medial surface of the obturator internus muscle and then protected with a malleable retractor. The ipsilateral femur was retracted laterally to facilitate exposure by pulling away the impacted femoral head on the acetabulum.

The pelvic ring fracture was fixed with a $3.5 \mathrm{~mm}$ reconstruction plate (Fig. 2) and the sacroiliac joint with two $7.5 \mathrm{~mm}$ cannulated screws under Image Intensifier guidance. The rectus sheath was repaired with continuous absorbable monofilament suture and the skin with interrupted nonabsorbable suture. Low-molecular weight heparin injections were continued postoperatively in all cases until the patients started ambulation. Radiographic measurement of residual displacement of the pelvic ring was measured from as the difference in the height of femoral head from a line perpendicular to the long axis of the sacrum. These results were then graded as excellent $(0-5 \mathrm{~mm})$, good $(6-10 \mathrm{~mm})$, fair $(11-15 \mathrm{~mm})$, and poor $(>15 \mathrm{~mm})^{4}$. Maximal articular surface displacement of the acetabulum was measured and graded as anatomic $(0-1 \mathrm{~mm})$, fair $(2-3 \mathrm{~mm})$, and poor $(>3 \mathrm{~mm})^{5}$.

Patients were examined at 2- week, 6- week, 3- month, 6month and one-year postoperatively. The modified Merle D' Aubigne and Postel Hip Scoring System was used to assess the functional outcomes. This scale consists of 3 domains related to the hip joint: pain, ambulation, and ROM. The

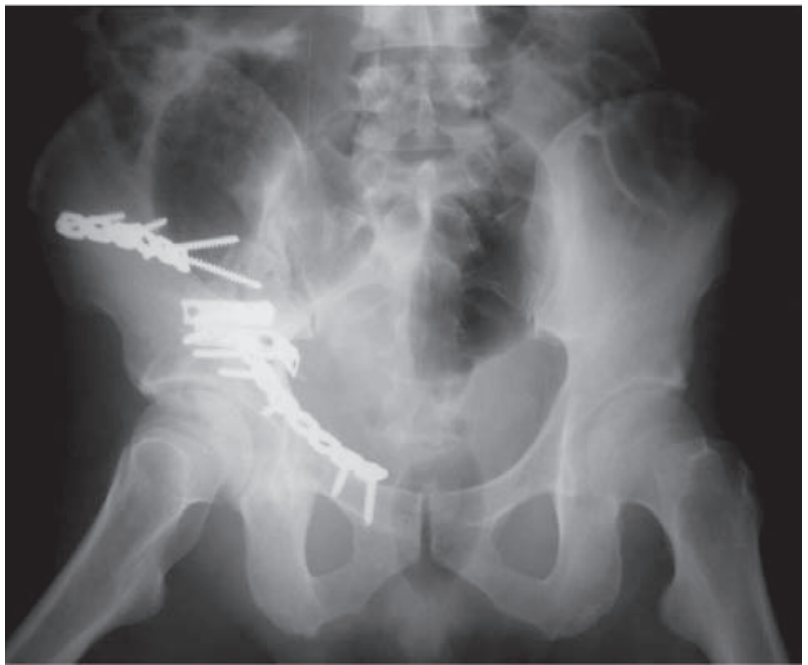

Fig. 2: Postoperative radiograph.

overall score was graded as excellent (18), good (15-17) fair (12-14), and poor (3-11).

\section{RESULTS}

All patients were male with a mean age of 37 years (range, 20 to 54y). Three patients were involved in motor vehicle accidents while the other 2 patients had fallen from a height. The mean Injury Severity Score (ISS) was 39 (range, 29 to 54). According to the AO Comprehensive Classification, there were four Type C1-2 pelvic injuries (sacroiliac dislocation or fracture dislocation) and one Type C1-3 injury (fracture of the sacrum). Three patients had concomitant acetabular fractures, one each of Type A3 (anterior column fracture), Type B2 (Tshaped fracture), and Type C3 (both column fracture) each.

The mean time lapse from injury to surgery was 9 days (range 0 to 22). An emergency surgery was performed on the day of trauma to fix one extremely unstable pelvic ring injury in a haemodynamically unstable patient. Surgery was delayed in another patient for 22 days due to failed conservative management. Two out of five patients had been previously treated with the external fixation. Mean operative time was 368 minutes (range, 220 to 510). All patients were transfused intraoperatively with a median of 4 units packed red cells. Blood loss totals were not available due to incomplete documentation. Two patients were admitted to the ICU postoperatively.

Two patients had preexisting foot drop before the surgery. One patient developed a superficial wound infection that resolved with antibiotic therapy, while another patient had seroma of the surgical wound. A bladder contusion was noted in a patient. Three patients had complains of postoperative erectile dysfunction. None experienced intraoperative peritoneal perforation or postoperative neurovascular complications. 
Excellent or anatomic reduction of the pelvic ring was achieved in 3 patients, and good reduction in 2 patients. Anatomic reduction was achieved in the 3 cases with the concomitant acetabular fractures. At the median follow up period of 12 months (range, 6 to $15 \mathrm{~m}$ ), functional score results were good in 3 patients, and fair in the 2 patients. Two patients experienced intermittent mild pelvic pain, while 3 patients had mild pelvic pain after ambulation. Four patients were able to walk without aid, one walked with crutches following postoperative management.

\section{DISCUSSION}

The modified Stoppa approach provides direct visualization of the entire pelvic brim from the pubic symphysis to the anterior aspect of the sacroiliac joint. Compared to the ilioinguinal approach, it is less invasive and does not require dissection of the inguinal canal, femoral vessels or femoral nerve. The lateral cutaneous nerve of the thigh is also well preserved. Another advantage of this approach is that fixation of the bilateral pelvic ring and acetabulum fractures can be performed through a single Pfannenstiel's incision ${ }^{6}$. Based on our experience, this approach results in better exposure for certain fracture types, such as medial wall fracture of the acetabulum. Similar to the ilioinguinal approach, the modified Stoppa approach is extra-articular and does not provide direct visualization of the posterior structures. Therefore, it should not be used in the fractures involving purely posterior structures, and in fractures more than 3 weeks old.

Selection of patients is of utmost importance when using this approach. All patients in the present study sustained at least an anterior column fracture; we managed to achieve excellent reduction in 3 patients and good reduction in 2 patients. No other approach was combined with the use of the modified Stoppa approach, except for one patient who underwent surgery 3 weeks after the trauma. In this case, a second posterior incision was necessary to break the callus and reduce the posterior column acetabular fracture. This case was later complicated by superficial wound infection, which fortunately resolved with antibiotic treatment.

An emergency surgery was performed in a haemodynamically unstable patient with pelvic ring injury caused by vertical shear force. The same patient had also sustained severe perineal injury and underwent perineal repair and colostomy procedures immediately after the pelvic fixation. All surgical wounds healed uneventfully. The colostomy was reversed 6 months after the injury. The patient was able to squat fully and ambulate without any aid after healing from the injury.
The mean operative time of 328 minutes in the present study was longer than other studies using a similar approach and the ilioinguinal approach ${ }^{2,5,6}$. The mean number of unit of red cells transfused was also higher than another study ${ }^{6}$. These differences can be explained by the fact that the Stoppa approach is relatively new among local surgeons and requires a rather steep learning curve. Secondly, all patients in this series had Type $\mathrm{C}$ unstable pelvic injuries compared to the other study, which also included type B injury.

Functional outcomes assessed using the modified Merle D' Aubigne system showed that 2 patients were rated good, and 3 were fair. Two patients had an incomplete lumbar sacral plexus injury and the other had a comminuted fracture of the olecranon process of the ulna preventing early ambulation. We also investigated urological and sexual dysfunction and found that 3 out of 5 patients had complaints of erectile dysfunction. The incidence of erectile dysfunction is extremely high compared with that of other studies 5,6,7. All patients in this study had either fracture disruption of the sacroiliac joint or fracture of the sacrum which may have injured the sacral plexus. Other potential causes of erectile dysfunction include perineal vascular injury, pudendal nerve injury, and post-traumatic stress.

A small number of patients had a relatively short period of follow presenting a limitation of this study. Nonetheless, we do know that none of the patients in the present study had postoperative complications such as post operative surgical wound hernia or intra operative peritoneal perforation that were attributable to use of the modified Stoppa approach,. Other complications which were not seen (but are commonly seen in the ilioinguinal approach) include femoral nerve and femoral vessel injuries, weakness of the hip adductor, and heterotrophic ossification. There was no avascular necrosis of the femoral head though longer follow up was necessary to definitively state there was no such occurrence.

\section{CONCLUSION}

Based on these preliminary results, the modified Stoppa approach offers another viable approach for unstable pelvic injury. It offers excellent visualization of the quadrilateral plate and anterior column, and in some cases the posterior column as well. It probably allows better reduction for certain types of unstable pelvic ring injury, especially for the protusio type or involvement of the quadrilateral plate. 


\section{REFERENCES}

1. Tile M, Helfet DL, Kellam JF. Fractures of the Pelvis and Acetabulum. Philadelphia: Lippincott Williams \& Wilkins. 2003: 13067.

2. Letournel E. The treatment of acetabular fractures through the iloinguinal approach. Clin Orthop Relat Res 1993; 62-76.

3. Hirvensalo E, Lindahl J, Bostman O. A new approach to the internal fixation of unstable pelvic fractures. Clin Orthop Relat Res 1993; 28-32.

4. Lindahl J, Hirvensalo E. Failure of reduction with an external fixator in the treatment of injuries of the pelvic ring: Long-term evaluation of 110 patients. J Bone Joint Surg [Br] 1999; 81: 955-62.

5. Matta J M, Tornetta III P. Internal fixation of unstable pelvic ring injuries. Clin Orthop Relat Res 1996; (369): 129-40.

6. Ponsen KJ, Joosse P, Schigt A, Goslings JC, Luitse JS. Internal fracture fixation using the Stoppa approach in pelvic ring and acetabular fractures: technical aspects and operative results. $J$ Trauma 2006; 61: 662-7.

7. Metze M, Tiemann AH, Josten C. Male sexual dysfunction after pelvic fracture. J Trauma 2007; 63(2): 394-401 